\title{
OCLUSÃO LINGUALIZADA PARA REABILITAÇÃO FINAL DE PACIENTE COM DISFUNÇÃO TEMPOROMANDIBULAR: RELATO DE CASO
}

\author{
COMPLETE DENTURES WITH LINGUALIZED OCCLUSION FOR REHABILITATION OF PATIENT WITH \\ TEMPOROMANDIBULAR DISORDER. CASE REPORT
}

\author{
Wagner Araújo de Negreiros* \\ Daniel Filgueiras Ferreira" \\ Rafael Leonardo Xediek Consani ${ }^{*+*}$ \\ Poliana Lima Bastos \\ Mayra de Maria Carvalho Mendes Ferreira
}

\begin{abstract}
RESUMO
Há mais de duzentos anos, diversas filosofias e técnicas de confecção das próteses totais vêm sendo discutidas, baseadas sobretudo em diferentes tipos de esquema oclusal e relacionamento dentário. A oclusão lingualizada é um tipo especial de padrão oclusal, caracterizado pelo contato de dentes anatômicos na arcada superior e dentes não-anatômicos na arcada inferior. Tal esquema pode representar um padrão de contatos oclusais que permitam menor quantidade de interferências oclusais, menor reabsorção óssea e, principalmente, maior liberdade de movimentação mandibular, sem travamento, condição esta favorável aos pacientes com sinais e sintomas temporomandibulares. O presente trabalho descreve a reabilitação oral de uma paciente por meio de próteses totais equilibradas com oclusão lingualizada. A paciente havia sido submetida à terapia com pistas deslizantes para tratamento de disfunçáo temporomandibular do tipo muscular. Os autores concluem que esse padrão oclusal pode ser bem indicado para pacientes disfuncionados por permitir adequada estabilidade da prótese pela redução das interferências e uma maior liberdade para movimentos funcionais da mandíbula.
\end{abstract}

DESCRITORES: Prótese Total • Oclusão dentária • Reabilitação oral.

\section{ABSTRACT}

Over the past of two hundred years, many philosophies and techniques of manufacturing the complete dentures have been discussed, based on different occlusal schemes and dental relationship. Lingualized occlusion is a special occlusal pattern characterized by contacts of anatomic teeth in the upper arch with non-anatomic teeth in the lower arch of the dentures. That scheme may present few occlusal interferences that promote a little bone resorption and freedom in the mandibular movement, and those conditions are favorable to patients with temporomandibular disorders. This paper reports a patient's mouth rehabilitation through double complete denture balanced by lingualized occlusion. The patient had been submitted to a slide plates therapy to treat a muscular disorder. The authors conclude that lingualized occlusion seems to be a well indicated occlusal pattern for patients with muscular disorders because it allows a satisfactory occlusal stability for reducing the occlusal interferences and freedom for the functional mandibular motion.

DESCRIPTORS: Denture, complete• Dental occlusion • Mouth rehabilitation

\footnotetext{
* Doutorando do Departamento de Prótese e Periodontia da Faculdade de Odontologia de Piracicaba (FOP-UNICAMP); Professor da Área de Prótese Dental da Universidade de Fortaleza (UNIFOR).

** Doutor em Clínica Odontologia pela Faculdade de Odontologia de Piracicaba (FOP-UNICAMP.)

*** Livre Docente, Professor da Área de Prótese Dental da Faculdade de Odontologia de Piracicaba (FOP-UNICAMP).

**** Doutora em Clínica Odontológica pela Faculdade de Odontologia de Piracicaba (FOP-UNICAMP).

***** Especialista em Saúde Pública pela Universidade de Ribeirão Preto-SP, pós-graduanda em Reabilitação Oral na Associação Brasileira de Cirurgião Dentista Piauí (ABCD).
} 
Negreiros WA, Ferreira DF, Consani RLX, Bastos PL, Ferreira MMCM. Oclusão lingualizada para reabilitação final de paciente com disfunção temporomandibular: relato de caso. Revista de Odontologia da Universidade Cidade de São Paulo 2009 mai-ago; 21(2): 185-8

\section{INTRODUÇÃO}

Oclusão em prótese total é um tema costumeiramente negligenciado entre cirurgióes-dentistas e técnicos em prótese dental. Muitas vezes, o clínico não atenta para as verdadeiras necessidades dos pacientes e delega todos esses importantes conceitos à atuação do técnico de laboratório.

Os principais objetivos para a utilização das próteses totais são a manutenção dos tecidos moles e duros dos arcos edêntulos, estética e função. De longa data, diversos estudos têm procurado definir um padrão de esquema oclusal que atinja realmente às necessidades dos pacientes, sem, contudo, chegarem a um consenso. Discute-se a respeito de se usar ou não uma oclusão balanceada, se esse balanceamento deve ser cêntrico e/ou excêntrico, que tipo de desoclusão é mais satisfatória, qual a melhor anatomia oclusal dos dentes artificiais, a quantidade ideal de dentes e de que maneira deve ser o arranjo desses no arco (Ortman ${ }^{4}$ 1977, Thomtson ${ }^{7}$ 1990, Dubojska et al. ${ }^{1}$ 1998).

Oclusão lingualizada é aquela em que existe o contato dentário em relação cêntrica apenas entre a cúspide lingual dos dentes maxilares com a fossa central dos dentes mandibulares (Parr e Ivanhoe ${ }^{5}$ 1996).

\section{DISCUSSÃO}

A oclusão lingualizada como padrão oclusal para próteses totais parece ser um conceito atual, entretanto, foi introduzido em 1927, por Alfred Gysi.

A anatomia dos dentes artificiais tem sido discutida. Um estudo realizado com quarenta nativos africanos observou que todos relataram comer todos os tipos de alimentos quando utilizando dentes anatômicos, enquanto que $87,5 \%$, só podiam comer alimentos moles quando utilizando dentes planos (Shetty ${ }^{6}$ 1964). Dentes com cúspides planas $\left(0^{\circ}\right)$ têm grande aceitação nos esquemas oclusais, na medida em que, apesar de possibilitarem uma menor eficiência mastigatória, consegue-se uma maior estabilização das dentaduras em função. É importante salientar que a utilização de dentes não-anatômicos diminui a quantidade de forças horizontais e oblíquas que causam maior reabsorção da crista óssea $\left(\right.$ Ortman $^{4}$ 1977). Jones ${ }^{2}$ (1972) ainda afirmou que os dentes sem cúspides permitem maior adaptabilidade a situaçóes de maloclusôes classes II e III, maior senso de liberdade, estando a mandíbula não travada em uma única posição, eliminação de forças horizontais, menor consumo de tempo para a técnica, mais conforto e eficiência em longo prazo.

Algumas escolas alemãs aconselham a utilização de dentes não-anatômicos associados a curvas de compensação, especialmente, em rebordos bastante reabsorvidos (Walter ${ }^{9}$ 1993). Um estudo afirmou que a forma ou anatomia dos dentes posteriores não é um fator significante no conforto do paciente (Woelsel et al. ${ }^{10}$ 1962). Por outro lado, a oclusão com dentes sem cúspides apresenta inconvenientes como a falta de penetração no alimento, estética insatisfatória e pouca estabilidade da mordida (Turbyfill $^{8}$ 1989).

\section{DESCRIÇÃO DO CASO CLÍNICO}

A paciente M.S.G.P., 63 anos, sexo feminino, aposentada, viúva, procurou tratamento na Clínica Odontológica da FOP - UNICAMP com o objetivo de substituir suas próteses totais e resolver uma condição dolorosa orofacial. A terapia convencional para disfunção temporomandibular foi inicialmente proposta, baseada na educação e orientação da paciente, fisioterapia, termoterapia e acompanhamento psicológico. Em relação ao componente oclusal, foi indicada a terapia com pistas deslizantes previamente à reabilitação final com próteses totais, conforme preconizado por Zuccolotto et al. ${ }^{11}$ (1999), a partir da modificação de próteses totais antigas (Ilustração 1). Após 4 meses de utilização das pistas deslizantes, observou-se a remissão da dor e dos demais sintomas relatados pela paciente (Negreiros et al. ${ }^{3}$ 2007).

A reabilitação final da paciente foi conduzida a partir da sequência convencional de procedimentos clínicos de confecção de próteses totais convencionais. $\mathrm{Na}$ fase de prova dos dentes fixados em cera, verificou-se adequada dimensão vertical de oclusão, conforto e aparência agradável

Posicionadas novamente no articulador semiajustável Dentatus, as próteses foram submetidas aos procedimentos de modificaçáo oclusal. Devido à dificuldade de se encontrarem dentes não-anatômicos no mercado odontológico, dentes semianatômicos convencionais foram transformados, por meio de desgaste com broca Maxicut, em dentes não-anatômicos.

Os contatos dentários foram marcados com carbono evidenciador para que as cúspides linguais dos dentes superiores tocassem a fossa central dos molares inferiores sem alteração da dimensão vertical de oclusão, caracterizando a oclusão lingualizada.

Apenas as cúspides vestibulares e linguais dos mola- 
Negreiros WA, Ferreira DF, Consani RLX, Bastos PL, Ferreira MMCM. Oclusão lingualizada para reabilitação final de paciente com disfunção temporomandibular: relato de caso. Revista de Odontologia da Universidade Cidade de São Paulo 2009 mai-ago; 21(2): 185-8

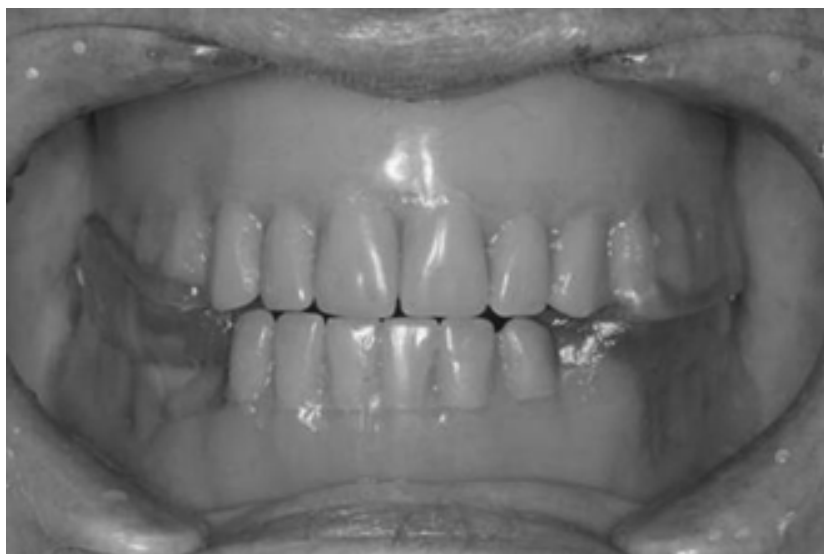

Ilustraçáo 1 Próteses totais antigas.

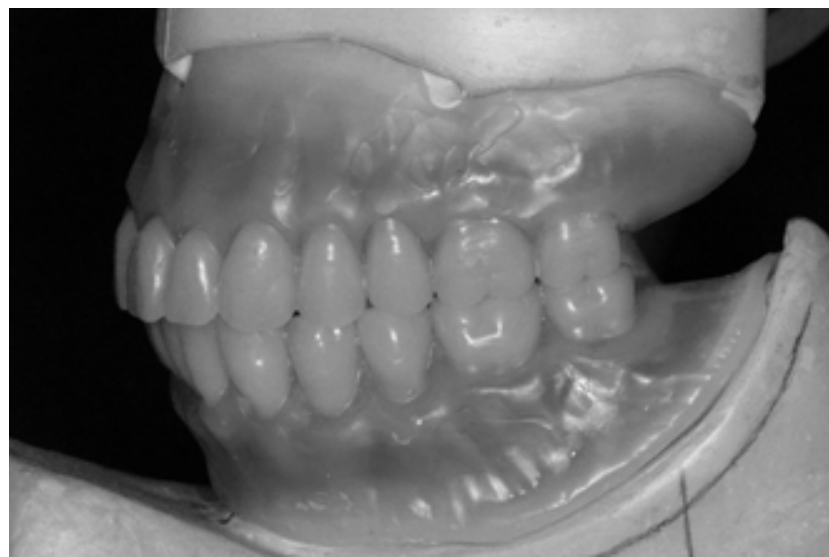

Ilustração 2 Adequada dimensão vertical de oclusão, conforto $e$ aparência agradável.

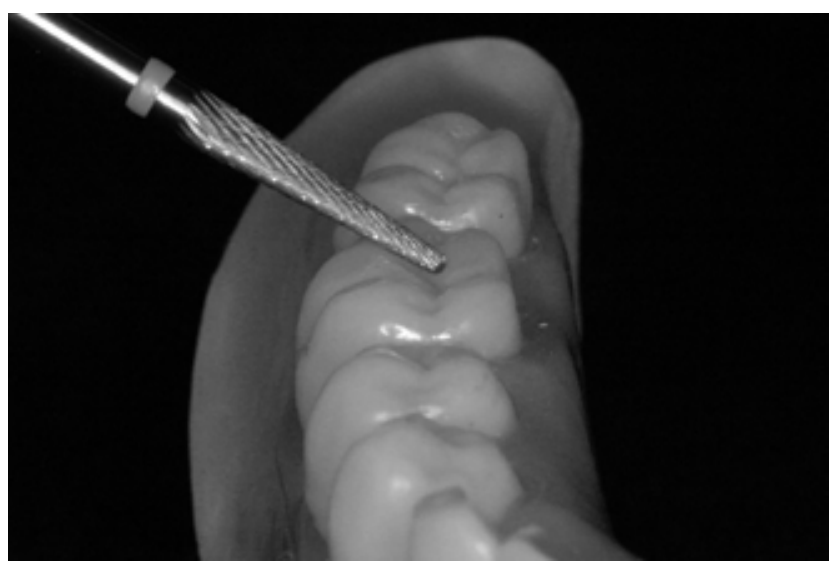

Ilustração 3 Desgaste com broca Maxicut em dentes nãoanatômicos.

res inferiores foram aplainadas e novamente as próteses foram experimentadas clinicamente.

Realizados os ajustes clínicos da prótese encerada, esta foi submetida aos procedimentos de inclusão, polimerização, acabamento e polimento.

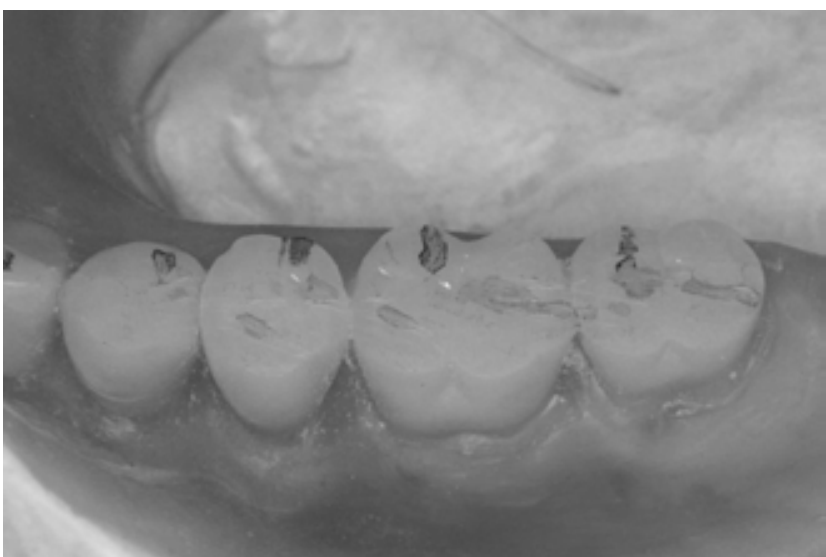

Ilustração 4 Dimensão vertical de oclusão, caracterizando a oclusão lingualizada.



Ilustraçáo 5 Próteses foram experimentadas clinicamente.



Ilustração 6 Procedimentos de inclusão, polimerização, acabamento e polimento.

O ajuste oclusal final foi uma etapa importante, permitindo liberdade de movimentação mandibular semelhante ao conseguido anteriormente com as pistas deslizantes.

$\mathrm{Na}$ fase final de instalação, verificou-se um relaciona- 
Negreiros WA, Ferreira DF, Consani RLX, Bastos PL, Ferreira MMCM. Oclusão lingualizada para reabilitação final de paciente com disfunção temporomandibular: relato de caso. Revista de Odontologia da Universidade Cidade de São Paulo 2009 mai-ago; 21(2): 185-8

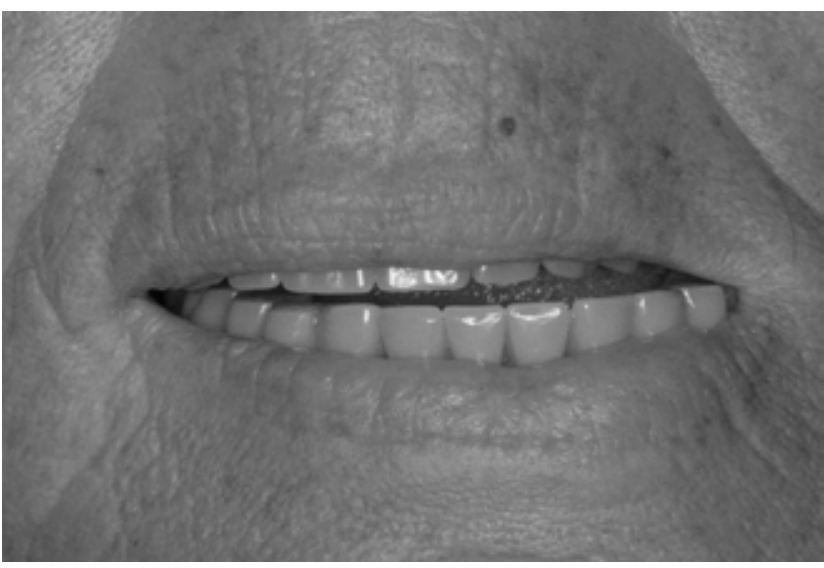

Ilustraçáo 7 Movimentação mandibular semelhante ao conseguido anteriormente com as pistas deslizantes.

mento oclusal semelhante ao anteriormente planejado, restaurando a função, o conforto e uma aparência estética agradável.

\section{CONCLUSÃO}

1) Existe grande discussão a respeito dos diversos conceitos da oclusão em prótese total, sobretudo quan-

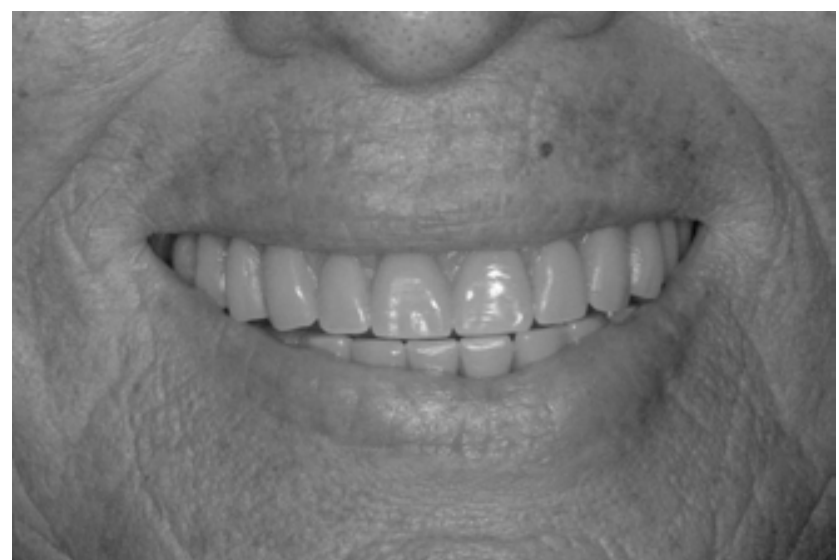

Ilustraçáo 8 Conforto e uma aparência estética agradável.

do se trata de esquemas oclusais e anatomia dos dentes artificiais;

2) O padrão de oclusão lingualizada pode apresentar vantagens de adequada estabilidade oclusal, menor reabsorção óssea dos rebordos alveolares e, principalmente, liberdade de movimentação mandibular, condiçóes clínicas favoráveis aos pacientes com sinais e sintomas temporomandibulares.

\section{REFERÊNCIAS}

1. Dubojska AM, White GE, Pasiek S. The importance of occlusal balance in the control of complete dentures. Quintessence Inter 1998 Jun; 29(6): 389-94.

2. Jones PM.The monoplane occlusion for complete denture. $J$ of American Dental Association 1972 Jul; 85(1): 49-100.

3. Negreiros WA, Ferreira DF, Consani RLX, Mesquita MF. Pistas deslizantes: uma alternativa terapêutica para pacientes com disfunção temporomandibular. Investigação (Franca), 2007; 6: 53-8.

4. Ortman HR. Complete denture occlusion. Dental Clin North Am 1977 Apr; 21(2): 299-320.

5. Parr GR, Ivanhoe JR. Lingualized occlusion: on occlusion for all reasons Dent Clin North Am 1996 Jan; 40(1): 103-12.

6. Shetty NS. Comparative observations of the use of cusp and zero-degree posterior teeth. J Prosthet Dent 1984 Apr; 51(4): 459-60.
7. Thompson H. Occlusion. 2nd. London: Wright; 1990.

8. Turbyfill WF. Regaining pleasure and success with complete denture services. Int J Prosthod 1989 SepOct; 2(5): 474-82.

9. Walter M. Compesating curves for non-anatomical teeth in complete denture construction. Eur J Prosthodont Restor Dent 1993 Sep; 2(1): 11-6.

10. Woelfel JB, Hickey JC, Allison ML. Effect of posterior tooth form on jaw and denture movement. $J$ Prosthet Dent 1962 Sep; 12(5): 922-39.

11. Zuccolotto MCC, Nóbilo KA, Nunes LJ, Hotta TH. Sliding plates on complete dentures as a treatment of temporomandibular disorder: a case report. Cranio, 1999 Oct; 17(4): 289-92.

Recebido em: 27/05/2008

Aceito em: 24/03/2009 